\title{
Peroxiredoxin 6 Mediates the Protective Function of Curcumin Pretreatment in Acute Lung Injury Induced by Serum From Patients Undergoing One- lung Ventilation in Vitro
}

Hui-Ting Li

Sun Yat-sen University Cancer Center

Fang Tan

the Seventh Affiliated Hospital of Sun Yat-sen University

Tian-Hua Zhang

Sun Yat-sen University Cancer Center

Long-Hui Cao

Sun Yat-sen University Cancer Center

Hong-ying Tan

Sun Yat-sen University Cancer Center

Wen-Qian Lin

Sun Yat-sen University Cancer Center

Wei-An Zeng

Sun Yat-sen University Cancer Center

Xin-Jin Chi ( $\nabla$ chixj@mail.sysu.edu.cn )

the Seventh Affiliated Hospital of Sun Yat-sen University

\section{Research Article}

Keywords: one-lung ventilation, lung injury, curcumin, peroxiredoxin 6, NF-kB signaling

Posted Date: December 3rd, 2021

DOI: https://doi.org/10.21203/rs.3.rs-1129709/v1

License: (c) (i) This work is licensed under a Creative Commons Attribution 4.0 International License.

Read Full License 


\section{Abstract}

Background: Curcumin has attracted much attention due to its wide range of therapeutic effects. In this study, we used serum collected from patients undergoing one-lung ventilation (OLV) to establish an in vitro acute lung injury (ALI) model to explore the potential protective mechanism of curcumin on ALI to provide a new reference for the prevention and treatment of ALI induced by OLV.

Methods: A549 cells were treated with $20 \%$ serum from patients undergoing OLV to establish an in vitro ALI model. Curcumin, at a dose of $40 \mu \mathrm{g} / \mathrm{ml}$, was administered two hours prior to this model. The levels of inflammation and oxidative stress markers were observed by Western blot, qRT-PCR, ELISA and reactive oxygen species assay. Additionally, the expression of peroxiredoxin 6 ( $\operatorname{Prdx} 6)$ and proteins involved in the NF-KB signaling pathway were evaluated.

Results: Twenty percent of serum collected from patients undergoing OLV downregulated the expression of Prdx6, leading to the activation of the NF-kB signaling pathway, which was associated with the subsequent overproduction of inflammatory cytokines and reactive oxygen species. Pretreatment with curcumin restored Prdx6 downregulation and inhibited NF-KB pathway activation by suppressing the nuclear translocation of P65, eventually reducing inflammation and oxidative stress damage in A549 cells.

Conclusions: Prdx6 mediated the protective function of curcumin by inhibiting the activation of the NF-KB pathway in ALI in vitro.

\section{Background}

One-lung ventilation (OLV) often results in mechanical ventilator-related lung injuries, which poses a great challenge to pulmonary function in patients undergoing chest surgery. The main causes of lung surgeryrelated mortalities have shifted from cardiac and surgical complications to pulmonary complications such as acute lung injury (ALI) [1]. Unlike other complications, the rate of ALI has not declined over the past decades [2, 3]. It is worth noting that pneumonectomy is associated with an increased risk of lung injury, and minor excision thoracic surgery may also result in similar pathology [4]. During thoracic surgery, the combined impacts of surgical procedures and OLV can lead to the release of proinflammatory factors and pro-oxidants that contribute to the development of $\operatorname{ALI}[5,6]$. Moreover, plasma collected from patients undergoing lobectomy or pneumonectomy has also shown evidence of oxidative damage [7]. Although the incidence of ALI caused by OLV remains high, there are currently no effective prevention methods. Therefore, it is particularly crucial to find effective therapeutic drugs.

Curcumin [1,7-bis(4-hydroxy-3-methoxyphenyl)-1,6-heptadiene-3,5-dione], a naturally occurring polyphenol extracted from the rhizome of turmeric, has gained much attention because of its pleiotropic pharmacological properties, such as anti-inflammatory [8], antioxidant [9], hypoglycemic [10], antibacterial [11] and immunomodulatory activities [12]. Newly synthesized metal-curcumin complexes have been shown to increase the solubility, cellular uptake and bioavailability of curcumin, improving its 
anti-inflammatory, antioxidant, antibacterial and antiviral effects and enhancing its clinical applications $[13,14]$. A number of studies have confirmed that curcumin has a protective effect on lung injury caused by a variety of pathological states that involve multiple regulatory mechanisms [15-17]. However, there are few studies on the role of curcumin in ALI induced by OLV and thoracic surgery.

Peroxiredoxin 6 ( $\operatorname{Prdx6}$ ) is the only mammalian 1-Cys member of the peroxiredoxin family. It has many functions, such as glutathione peroxidase (Gpx) activity, calcium-independent phospholipase A2 (aiPLA2) activity, and lysophosphatidylcholine acyltransferase (LPCAT) activity. It has been shown to be involved in redox homeostasis, phospholipid turnover, glycolipid metabolism, and cell signal transduction. Existing research has indicated that Prdx6 is closely related to the occurrence and development of ALI. In a lipopolysaccharide (LPS)-induced lung injury mouse model, inactivation of the aiPLA2 activity of Prdx6 was associated with reduced mortality and prevention of lung inflammation and oxidative stress [18]. Similarly, Yang et al. found that deletion of Prdx6 exaggerated LPS-induced ALI with increased oxidative stress in vivo [19]. These results suggests that Prdx6 may play an important role in ALI induced by OLV, and further studies are needed to test our hypothesis.

In the present study, we first established an in vitro model of ALI induced by intervention of serum collected from patients undergoing OLV and then used this model to explore the effect of pretreatment with curcumin. Finally, we explored the underlying regulatory mechanisms involved.

\section{Materials And Methods}

\section{Patients and methods}

From February 1, 2021, patients who underwent video-assisted thoracoscopic surgery (VATS) for lung cancer at the Sun Yat-Sen University Cancer Center (SYSUCC; Guangzhou, China) were assessed for eligibility. Exclusion criteria included pneumonectomy and thoracotomy. Written informed consent was obtained from all patients. The study protocol was approved by the ethics committee of SYSUCC.

\section{Anesthetic procedure, surgery and blood sample collection}

Anesthetic procedures were standardized for all patients to minimize the impact of anesthesia differences between patients, with the use of double-lumen bronchial catheterization and positioning with fiberoptic bronchoscopy to ensure OLV. Intravenous anesthesia induction was applied using bolus doses of fentanyl, cisatracurium and propofol. Intravenous inhalation combined with anesthesia was applied intraoperatively using continuous infusions of remifentanil, cisatracurium and propofol and continuous inhalation of sevoflurane.

A protective ventilation strategy was applied during OLV. Briefly, a low tidal volume of $\sim 6-7 \mathrm{ml} / \mathrm{kg}$ body weight and a $3-5 \mathrm{~cm} \mathrm{H}_{2} \mathrm{O}$ of positive end-expiratory pressure were applied, with the peak airway pressure limited below $30 \mathrm{~cm} \mathrm{H}_{2} \mathrm{O}$. The concentration of inhaled oxygen in the ventilated lung fluctuated from $80-$ 
$100 \%$ and was adjusted according to repeated arterial blood gas analysis. If pulse oxygen saturation $\left(\mathrm{SpO}_{2}\right)$ fell below $80 \%$, then high-frequency ventilation equipment was used. VATS-assisted lobectomy was performed by three thoracic surgeons in the same medical group using similar surgical techniques. Central venous blood samples were obtained from the internal jugular vein before OLV and before the end of OLV, and were marked as OLV before and OLV $\mathrm{Ofter}_{\text {. }}$ After placement for 30 minutes at room temperature, the entire blood samples were centrifuged at $3000 \mathrm{bpm}$ for 15 minutes. Then, the serum in the upper layer was collected and preserved at $-80^{\circ} \mathrm{C}$.

\section{Cells and reagents}

The human alveolar epithelial cell line A549 was cultured in Dulbecco's S modified Eagle's medium (DMEM, Gibco, USA) containing 10\% fetal bovine serum (FBS, HyClone, USA) and $1 \%$ penicillinstreptomycin (Thermo Fisher Scientific, USA). The cells were incubated at $37{ }^{\circ} \mathrm{C}$ in a humidified $5 \% \mathrm{CO}_{2}$ incubator. Curcumin (78246-100 mg, Sigma-Aldrich, USA) was dissolved in dimethyl sulfoxide (DMSO, D2650-100 mL, Sigma-Aldrich, USA) and diluted with complete medium. Small interfering RNA (siRNA) targeting Prdx6 and scrambled RNA (NC-siRNA) controls were purchased from Kidan Biosciences (Guangzhou, China). The sequence and antisequence of Prdx6 siRNA-1, siRNA-2 and siRNA-3 were 5'GACAGUGUUGAGGACCAUCUUTT-3' and 5'-AAGAUGGUCCUCAACACUGUCTT-3'; 5'CCGAAAGGAGUCUUCACCAAATT-3' and 5'-UUUGGUGAAGACUCCUUUCGGTT-3'; and 5'CGCAUCCGUUUCCACGACUUUTT-3' and 5'-AAAGUCGUGGAAACGGAUGCGTT-3', respectively. The transfection process was carried out using Lipofectamine 3000 reagent (Carlsbad, Invitrogen, USA) according to the manufacturer's instructions.

\section{Experimental design}

4.1 Serum concentrations from patients undergoing OLV induce lung inflammation and oxidative stress.

To verify whether the serum from patients undergoing OLV could induce lung injury and to explore the appropriate concentration of OLV serum, the cells were treated with FBS-free DMEM with or without serum from patients undergoing OLV for $48 \mathrm{~h}$ when $\mathrm{A} 549$ cells reached $70 \%-80 \%$ confluence. The groups were set as follows: (1) control group: only the same volume of FBS-free DMEM was added, (2) $10 \%$ OLV $_{\text {before }}$ serum: FBS-free DMEM containing $10 \%$ serum from patients before OLV, (3) $20 \%$ OLV $_{\text {before }}$ serum: FBS-free DMEM containing $20 \%$ serum from patients before OLV, (4) $30 \%$ OLV $_{\text {before }}$ serum: FBSfree DMEM containing $30 \%$ serum from patients before OLV, (5) $10 \%$ OLV $_{\text {after }}$ serum: FBS-free DMEM containing $10 \%$ serum from patients after OLV, (6) $20 \%$ OLV $_{\text {after }}$ serum: FBS-free DMEM containing $20 \%$ serum from patients after OLV, and (7) $30 \%$ OLV $_{\text {after }}$ serum: FBS-free DMEM containing $30 \%$ serum from patients after OLV.

After $48 \mathrm{~h}$ of intervention, cell morphology was observed with an electric fluorescence microscope (Nikon ECLIPSE Ti2, Nikon, Japan). The expression of IL- 6 at the protein and gene levels was detected by Western blot and qRT-PCR (quantitative reverse transcription PCR) in A549 cells. In addition, to test 
reactive oxygen species (ROS) levels, an ROS assay was performed using dichlorodihydrofluorescein diacetate (DCFH-DA) staining according to the manufacturer's instructions.

\subsection{Dosage of curcumin for reducing $\mathrm{OLV}_{\text {after }}$ serum-induced lung injury.}

To explore the appropriate dose of curcumin pretreatment in the A549 cell line, which can affect OLV serum-induced lung injury without harming the cells, we first preprocessed the A549 cells with three different concentrations $(40,80$ and $160 \mu \mathrm{g} / \mathrm{ml}$ ) of curcumin for $2 \mathrm{~h}$. Curcumin was dissolved in DMSO on the day of the experiment and diluted in serum-free medium to ensure that the final concentration of DMSO was $<0.05 \%$, and the same concentration of DMSO was used in the control group. Then, according to the results based on the previous sections, $20 \%$ OLV $_{\text {after }}$ serum ( $20 \%$ serum from patients after OLV) was added to induce lung inflammation and oxidative stress injury. After the cells were treated with $20 \%$ $\mathrm{OLV}_{\text {after }}$ serum for $48 \mathrm{~h}$, the expression of IL- 6 and the level of ROS were assessed by Western blot, qRTPCR and DCFH-DA staining.

4.3 The effect of curcumin pretreatment on $\mathrm{OLV}_{\text {after }}$ serum-induced inflammation and oxidative stress injury in A549 cells.

Here, we pretreated A549 cells with $40 \mu \mathrm{g} / \mathrm{ml}$ curcumin (the dose of curcumin was determined according to the results obtained in the previous section) for $2 \mathrm{~h}$. Then, after $2 \mathrm{~h}$, the culture medium containing curcumin was discarded, and the cell surface was thoroughly washed three times with PBS. Next, the A549 cells were incubated with FBS-free DMEM containing $20 \%$ serum from patients after OLV for $48 \mathrm{~h}$. The cells and cell culture medium were harvested for subsequent inflammation and oxidative stress factor measurements. ROS was detected by DCFH-DA staining. Meanwhile, the expression of Prdx6 was tested by qRT-PCR, Western blot and immunofluorescence.

4.4 The role of Prdx6 in the protective effects of curcumin pretreatment on serum from OLV patientinduced lung injury and its related mechanism.

To explore the potential mechanism of the protective effect of curcumin, the expression of proteins involved in the NF-KB signaling pathway was analyzed. To verify the relationship between Prdx6 and the NF-KB signaling pathway in vitro, we used siRNA to investigate the function of Prdx6 in the protective effects of pretreatment with curcumin on serum from OLV patient-induced lung injury.

First, we transfected A549 cells with Prdx6-siRNA or NC-siRNA and verified its effects by Western blot and qRT-PCR. Second, siRNA-transfected cells were pretreated with $40 \mu \mathrm{g} / \mathrm{ml}$ curcumin for $2 \mathrm{~h}$, and an inflammation and oxidative stress injury model induced by $20 \% \mathrm{OLV}_{\text {after }}$ serum was established, and NCsiRNA was used as a control. Then, inflammatory oxidative stress factors and the production of ROS, as well as NF-kB pathway-related proteins, were detected. Furthermore, the expression of P65 was analyzed by immunofluorescence, and the Proteolytic Nuclear and Cytoplasmic Protein Extraction kit (Cat. No: P5103, NCM) was used to extract cytoplasmic protein and nucleoproteins from the treated cells. In 


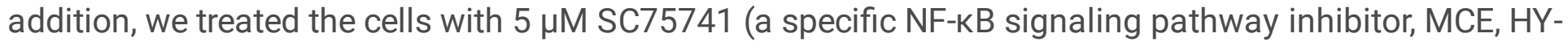
10496) for $48 \mathrm{~h}$ to delineate the detailed mechanism.

\section{Experimental method \\ 5.1 qRT-PCR}

An RNA rapid extraction kit (EZBioscience, China) was used to extract total RNA from the treated A549 cells according to the manufacturer's instructions. The concentration and purity of extracted RNA were analyzed by a Nanodrop 2000 (Thermo Fisher Scientific, USA). cDNA was prepared using the RevertAid First Strand cDNA Synthesis kit (Thermo Fisher Scientific, USA). The qRT-PCR protocol was set as $95{ }^{\circ} \mathrm{C}$ for 2 minutes, followed by 40 cycles of 10 seconds at $95^{\circ} \mathrm{C}$ and 30 seconds at $60{ }^{\circ} \mathrm{C}$. The primers were designed as follows: IL-6 (forward, 5'-AGACAGCCACTCACCTCTTCAG-3'; reverse, 5'-

TTCTGCCAGTGCCTCTTTGCTG-3'); peroxiredoxin 6 (forward, 5'-CAGCTACCACTGGCAGGAACTT-3'; reverse, 5'-GGAAGGACCATCACACTATCCC-3'); and GAPDH (forward, 5'-GTCTCCTCTGACTTCAACAGCG-3'; reverse, 5'-ACCACCCTGTTGCTGTAGCCAA-3'). The expression levels of the target genes were normalized to the levels of GAPDH as an endogenous control in each group. The relative fold expression level of mRNAs was calculated using the $2^{-\triangle \Delta C t}$ method.

\subsection{Immunoblotting}

Total protein lysates from cells were loaded on SDS-PAGE gels for electrophoretic separation and transferred onto polyvinyl difluoride membranes, blocked with $5 \%$ milk and probed with the following primary antibodies: anti-IL-6 (absin; \#abs135607; 1:1000), anti-IL-1 $\beta$ (absin; \#abs235771; 1:1000), anti-IL10 (absin; \#abs136414; 1:1000), anti-TNF-a (CST; \#3707S; 1:1000), anti-Peroxiredoxin 6 (Abcam; \#ab133348; 1:1000), anti-phospho-IKKa/ $\beta$ (CST; \#2697S; 1:1000), anti-phospho-IKB alpha (Affinity; \#AF2002; 1:1000), anti- IKB alpha (Affinity; \#AF5002; 1:1000), anti-NF-KB P50 (CST; \#3035; 1:1000), anti-

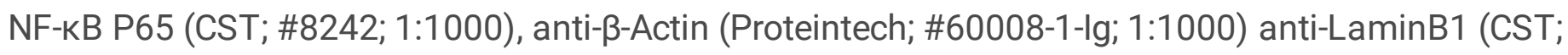
$\# 13435 ; 1: 1000)$ and anti-GAPDH (CST; \#2118S; 1:1000). On the second day, the membranes were incubated with HRP-conjugated secondary antibodies (Abcam; goat anti-rabbit IgG, goat anti-mouse IgG; 1:10000). Subsequently, an enhanced chemiluminescence solution was used to detect the immunocomplexes. The relative quantification of protein was measured using ImageJ software.

\subsection{Reactive oxygen species (ROS) assay}

ROS generation was evaluated using 2',7'-dichlorofluorescin diacetate (DCFH-DA, D6883, Sigma-Aldrich, USA) staining. In simple terms, the cells were incubated with $10 \mu \mathrm{M} \mathrm{DCFH-DA}$ for 30 min at $37^{\circ} \mathrm{C}$ in the dark. Then, the stained cells were washed three times with PBS. A detected signal with an excitation wavelength of $488 \mathrm{~nm}$ and an emission wavelength of $525 \mathrm{~nm}$ by a fluorescence microscope (Nikon ECLIPSE Ti2, Nikon, Japan) was used. The fluorescence intensity of DCF-DA was normalized to a negative control.

\subsection{Enzyme-linked immunosorbent assay (ELISA)}


The levels of superoxide dismutase (SOD, MM-0390H2, Meimian Industrial Co., China) and malondialdehyde (MDA, JL11466, J\&L Biological, China) in the cell culture supernatant were quantified using ELISA kits. All indices were analyzed by the same technician according to the manufacturer's instructions.

\subsection{Immunofluorescent staining}

The cells were washed three times with PBS, fixed with $4 \%$ paraformaldehyde and permeabilized with $0.1 \%$ Triton $\mathrm{X}-100$ in PBS. After blocking with $5 \%$ bovine serum albumin (BSA) in $\mathrm{dd}_{2} \mathrm{O}$ for 40 min at room temperature, the cells were incubated with a diluted primary antibody (anti-peroxiredoxin 6, Abcam, \#ab133348, 1:100; anti-NF-KB P65, CST, \#8242, 1:100) at $4^{\circ} \mathrm{C}$ overnight, followed by secondary antibody incubation. Cell nuclei were stained with DAPI (Thermo Fisher Scientific, P36966, $20 \mu \mathrm{g} / \mathrm{mL}$ ).

Representative images were acquired using a confocal microscope image system (OLYMPUS FV1000, OLYMPUS, Japan).

\section{Statistical analysis}

Statistical analyses were conducted using GraphPad Prism 7 (GraphPad Software, Inc., La Jolla, CA, USA) software. Comparisons between the two groups and among multiple groups were performed using two-sided $t$ tests and one-way ANOVA, respectively. All data are expressed as the mean \pm standard deviation (SD). A $P$ value $<0.05$ was considered statistically significant.

\section{Results}

\section{Twenty percent $O L V_{\text {after }}$ serum induced the most obvious inflammation and oxidative stress in A549 cells.}

After $48 \mathrm{~h}$ of intervention with $10 \%, 20 \%$, and $30 \%$ OLV $_{\text {before }}$ serum or OLV $_{\text {after }}$ serum in A549 cells, the cell morphology was observed with a microscope in each group. As shown in Fig. $1 \mathrm{~A}$, when compared with the control group, in both the $O L V_{\text {before }}$ serum and $O L V_{\text {after }}$ serum intervention groups, with the increase in serum concentration, the cell density per unit area gradually decreased, while the number of irregular cells gradually increased. Then, the expression of IL- 6 at the protein and gene levels was detected by Western blot and qRT-PCR, respectively. The results indicated that $20 \% \mathrm{OLV}_{\text {after }}$ serum could induce the highest level of IL- 6 among all the groups, at both the protein and genetic levels, and produces the most obvious inflammatory response in A549 cells (Fig. 1B, 1C and 1D). In addition, the ROS assay showed similar results, which confirmed that $20 \% \mathrm{OLV}_{\text {after }}$ serum could induce a significant oxidative stress response (Fig. 1E).

\section{Pretreatment with $40 \mu \mathrm{g} / \mathrm{ml}$ curcumin in A549 cells is a suitable concentration to alleviate OLV serum- induced inflammation and oxidative stress.}

A549 cells were pretreated with different concentrations of curcumin for $2 \mathrm{~h}$, followed by $48 \mathrm{~h}$ of $20 \%$ $\mathrm{OLV}_{\text {after }}$ serum intervention. Then, the expression of IL- 6 and the level of ROS were assessed. As shown in 
Fig. 2A, 2B and 2C, 20\% OLV after serum significantly increased the level of IL-6 at both the protein and gene levels. Compared with the control group, pretreatment with $40 \mu \mathrm{g} / \mathrm{ml}, 80 \mu \mathrm{g} / \mathrm{ml}$ and $160 \mu \mathrm{g} / \mathrm{ml}$ curcumin alone without serum intervention slightly increased IL-6 levels, but no significant difference was observed. Compared with the serum intervention group, pretreatment with curcumin at $40 \mu \mathrm{g} / \mathrm{ml}$ and 80 $\mu \mathrm{g} / \mathrm{ml}$ in serum intervention cells reduced the protein level of IL-6 most significantly. In the ROS assay, the results indicated that $20 \% \mathrm{OLV}_{\text {after }}$ serum could cause an obvious oxidative response. After pretreatment with curcumin, the production of ROS decreased significantly, and the reduction level of ROS in the three curcumin treatment groups was similar (Fig. 2D). Based on the above results, to minimize additional drug damage to cells, we chose the lower concentration $(40 \mu \mathrm{g} / \mathrm{ml})$ of curcumin for the following experiments.

\section{Curcumin pretreatment reduced OLV serum-induced inflammation and oxidative stress and could be related to Prdx6.}

Pretreatment with $40 \mu \mathrm{g} / \mathrm{ml}$ curcumin was used to intervene in the inflammation and oxidative stress induced by $20 \%$ OLV $_{\text {after }}$ serum in A549 cells and to explore the effect of curcumin on the injury induced by serum collected from patients undergoing OLV in alveolar epithelial cells. We detected the protein expression of classic proinflammatory and anti-inflammatory cytokines, such as IL-6, IL-1 $\beta$, TNF-a and IL10 , by Western blot. At the same time, the expression of MDA and SOD in the cell culture supernatant was quantified using an ELISA kit. As shown in Fig. 3A, 3B and 3C, serum from patients undergoing OLV' stimulation produced obvious inflammatory and oxidative reactions, mainly manifested by an increase in expressions of IL-6, IL-1 $\beta$, TNF- $\alpha$ and MDA and a decrease in expressions of IL-10 and SOD. Pretreatment with curcumin reversed these changes. Similarly, the results of the ROS assay also confirmed that curcumin could significantly reduce the increase in ROS content induced by OLV serum (Fig. 3D). Furthermore, we observed the expression of Prdx6 in each group by Western blot, qRT-PCR and immunofluorescence and found that OLV serum could significantly reduce the expression of Prdx6, while pretreatment with curcumin could reverse it (Fig. 3E, 3F and 3G).

\section{Prdx6 in the protective effects of curcumin pretreatment on serum from OLV patient-induced injury and association with the NF-KB pathway.}

To explore the potential mechanism of the protective effect of curcumin, we examined the proteins involved in the NF-KB pathway. After OLV serum intervention, the expression of P-IKKa/ $\beta$ and P-IKBa was increased significantly, while that of IKBa was decreased. When curcumin was used, the expression of $P$ $\mathrm{IKKa} / \beta$ and P-IKBa was significantly decreased, while that of IKBa was increased (Fig. 4A). To verify the relationship between Prdx6 and the NF-kB signaling pathway, we transfected A549 cells with Prdx6-siRNA or NC-siRNA. As shown in Fig. 4B and 4C, Prdx6-siRNA significantly reduced the expression of Prdx6 at both the protein and gene levels. Among them, Prdx6-siRNA 2 and siRNA 3 possessed the most obvious reduction effect. Therefore, we used Prdx6-siRNA 2 or siRNA 3 to transfect A549 cells. The transfected cells were pretreated with $40 \mu \mathrm{g} / \mathrm{ml}$ curcumin for $2 \mathrm{~h}$, and an inflammation and oxidative stress injury model induced by $20 \%$ OLV $_{\text {after }}$ serum was established. Compared with that in the NC-siRNA group, the expression of pro-inflammatory factors, including IL-6, IL-1 $\beta$ and TNF-a, in the Prdx6-siRNA group was 
significantly increased and anti-inflammatory IL-10 markedly reduced (Fig. 4D). ROS assays demonstrated that ROS generation in the Prdx6-siRNA group was significantly higher than that in the NCsiRNA group (Fig. 4E). Our ELISA results further confirmed that when compared with the NC-siRNA group, the expression of the pro-oxidative stress factor MDA significantly increased, while the expression of the antioxidative stress factor SOD obviously decreased in the Prdx6-siRNA group (Fig. 4F). Subsequently, we detected NF-KB pathway-related proteins in these two groups and found that, compared with the NCsiRNA group, the expression of $\mathrm{P}-\mathrm{IKKa} / \beta$ and $\mathrm{P}-\mathrm{IKBa}$ was significantly increased, while the expression of IKBa was slightly decreased in the Prdx6-siRNA group (Fig. 4G).

\section{Prdx6 inhibits activation of the NF-KB signaling pathway by suppressing the nucleus translocation of P65 to participate in curcumin pretreatment on serum from OLV patient-induced inflammatory damage.}

NC-siRNA- or Prdx6-siRNA-transfected A549 cells were pretreated with $40 \mu \mathrm{g} / \mathrm{ml}$ curcumin for $2 \mathrm{~h}$, and an inflammation and oxidative stress injury model induced by $20 \%$ OLV $_{\text {after }}$ serum was established. Immunofluorescence staining was performed, and the ProtLytic Nuclear and Cytoplasmic Protein Extraction kit was used to extract the cytoplasmic protein and nucleoprotein of the two groups to detect P65 expression in the treated cells. The results of the two detection methods in Fig. 5A and 5B showed that P65 was in both the cytoplasm and nucleus of curcumin-pretreated A549 cells. Additionally, compared to the NC-siRNA group, the expressions of P50 and P65 in the nucleus of the Prdx6-siRNA group were significantly higher, suggesting that Prdx6 suppressed P65 and P50 translocation into the nucleus to inhibit activation of the NF-KB signaling pathway. To further confirm the role of the NF-KB signaling pathway in the protective effect of curcumin pretreatment on serum from OLV patient-induced inflammatory damage, we performed a rescue assay using SC75741, which is a specific NF-KB inhibitor. As shown in Fig. 4D, SC75741 attenuated inflammatory damage induced by $20 \%$ OLV $_{\text {after }}$ serum in A549 cells, as determined by inhibiting the expression of proinflammatory factors such as IL-6, IL-1 $\beta$ and TNF-a and increasing the levels of anti-inflammatory IL-10.

In summary, curcumin preconditioning exerted a protective effect on lung injury induced by serum from OLV patients in A549 cells by increasing the expression of Prdx6. On the one hand, Prdx6 can reduce the inflammatory response by suppressing the nuclear translocation of P65 to inhibit activation of the NF-KB signaling pathway, and on the other hand, Prdx6 can also reduce oxidative stress injury by increasing the activity of antioxidant enzymes and inhibiting the activity of pro-oxidation factors.

\section{Discussion}

In this study, we simulated acute lung injury caused by OLV at the cellular level for the first time, which can be used in future relevant research models. Here, we showed that the application of $20 \% \mathrm{OLV}_{\text {after }}$ serum could induce obvious inflammation and oxidative stress in A549 cells. We further found that 40 $\mu \mathrm{g} / \mathrm{ml}$ curcumin pretreatment could significantly alleviate OLV serum-induced inflammation and oxidative stress and was related to Prdx6 regulation. 
As a special mode of mechanical ventilation, OLV is a highly complex procedure and plays a key role, especially in thoracic surgery. However, like a double-edged sword, it can also be harmful to patients due to ventilator-induced lung injury caused by various mechanisms [20]. During the period of OLV in lung surgery, despite the normalization of hemodynamic and ventilatory patterns, the ventilation/perfusion (V/Q) mismatch still exists in both the dependent and nondependent lungs, which has more damaging consequences than does a period of complete lung collapse and surgical manipulation [21]. In addition, according to the "multiple-hit hypothesis," some researchers regard lung injury caused by two-lung ventilation in thoracic surgery as the first hit. The second hit is caused by OLV and surgical procedures. Alveolar recruitment and accompanying re-expansion/reperfusion-induced lung injury are considered the third hit [22]. Multiple damage factors work together to induce lung injury, characterized by diffuse epithelial and endothelial injury with infiltration of inflammatory cells, recruitment of leukocytes to the lung and increased lung permeability, resulting in lung edema, surfactant dysfunction and deterioration of pulmonary gas exchange. Proinflammatory cytokines such as IL-6, IL-1 $\beta$ and TNF-a play crucial roles in inflammation. Among them, IL- 6 is a major player in cytokine storms, and increased levels of IL- 6 are associated with the severity of acute inflammation. In addition, the serum levels of IL- 6 and TNF- $a$ are known to be significant predictors of disease severity and death $[23,24]$. Therefore, IL- 6 was selected as an indicator of the inflammatory response in our study. Moreover, the lungs are major organs of systemic and pulmonary oxidative stress. Clinical and experimental studies have shown that OLV in thoracic surgery is a powerful free radical generator $[25,26]$. The excessive production of ROS has been proven to be related to the decline of the antioxidant defense system during aging and/or the decline of the antioxidant defense system in cells facing environmental or cellular stress, resulting in the failure of intracellular homeostasis [27]. Oxidative stress caused by this redox imbalance leads to the pathogenesis of a variety of lung diseases, such as ALI and acute respiratory distress syndrome (ARDS) [28, 29]. MDA is a good indicator of free radical activity, and its elevation represents increased lipid peroxidation. SOD is an endogenous antioxidant enzyme that can detoxify free radicals. According to our results, $20 \% \mathrm{OLV}_{\text {after }}$ serum from thoracic surgery patients could induce a significant inflammation and oxidative stress response in vitro, which is characterized by an obvious increase in IL-6 and ROS expressions.

Recently, curcumin has attracted much attention due to its anti-inflammatory, antioxidant, antitumor and other pharmacological effects. Many studies have revealed that curcumin has a protective effect by increasing the expression of endogenous Prdx6 [30-32]. Prdx6 is a 1-cysteine PRDX, a multifunctional enzyme with not only Gpx and aiPLA2 but also LPCAT activity. Prdx6 may reduce aggregate-induced oxidative toxicity via its Gpx activity; in turn, it is possible to regulate inflammation/immune networks and mitochondrial oxidative stress by interacting with pathogenic genes and encouraging toxic protein aggregation through its aiPLA2 activity [33]. Prdx6 is closely related to the occurrence and development of ALI, and it has been shown that Prdx6 deficiency initiates ROS-induced endoplasmic reticulum stress that leads to cell death [34]. Fisher's team found that the inactivation of peroxiredoxin 6 phospholipase A2 activity can protect against lung injury in mouse models of ventilator-induced lung injury and LPSinduced ALI [18, 35]. Moreover, many studies have shown that Prdx6 knockout aggravates LPS-, cecal ligation- and puncture-induced ALI by augmenting inflammation, oxidative stress and matrix degradation 
$[18,36]$. In our research, $20 \%$ OLV $_{\text {after }}$ serum decreased the expression of Prdx6 in A549 cells, and curcumin pretreatment not only obviously reversed the expression of Prdx6 but also reduced the increased levels of inflammatory factors and reactive oxygen free radicals to reduce inflammation and oxidative stress injury, which was consistent with results reported in other studies [30, 31]. Subsequently, we used siRNA to verify the relationship between Prdx6 and lung injury and found that Prdx6-siRNA aggravated inflammation and oxidative stress induced by serum from patients undergoing OLV. Our results suggests that Prdx6 is essential for cellular protection. A deficiency in Prdx6 not only increased the production of proinflammatory factors and oxygen free radicals but also decreased the expression of anti-inflammatory factors and antioxidant enzymes. In our model of lung injury induced by OLV in vitro, curcumin pretreatment inhibited inflammation and oxidative stress to protect cells by stimulating endogenous Prdx6 production.

Nuclear factor kappa B (NF-kB) signaling regulates important physiological processes, such as inflammation, immune responses, cell survival and cancer. When cells are stimulated, the IKK complex (IKKa, IKKß and IKKY) in the cytoplasm is rapidly activated by proinflammatory signaling cascades, and then IKBa is phosphorylated and recognized by ubiquitinating enzymes. Subsequently, the resulting proteasomal degradation of IKB proteins releases IKB-bound NF-KB transcription factors (P50 and P65), which are transferred to the nucleus to drive the expression of target genes [37]. There is evidence that Prdx6 plays its biological role by regulating the NF-KB signaling pathway [31, 38, 39]. Prdx6 interrupts the formation of the TRAF6-ECSIT complex induced by TLR4 stimulation, leading to suppression of bactericidal activity by inhibiting NF-KB activation in the cytoplasm [39]. In addition, Prdx6 also plays a protective role against radiation by interacting with the TLR4 receptor and subsequently activating the NF-KB signaling pathway to trigger cellular defense mechanisms [38]. In the present study, curcumin pretreatment restored Prdx6 downregulation, which in turn normalized NF-KB signaling. Further application of SC75741, an inhibitor of NF-KB, reduced the effect of Prdx6-siRNA on the OLV seruminduced inflammatory response, suggesting involvement of the NF-KB signaling pathway in the protective effects of Prdx6. As with our results, Chhunchha et al. found that curcumin could attenuate endoplasmic reticulum stress and NF-kB-mediated abnormal signaling to reduce hypoxia-induced oxidative stressmediated cell death in mouse hippocampal cells (HT22) by increasing Prdx6 expression [31]. We further investigated the specific mechanism by which Prdx6 regulates the NF-KB signaling pathway by detecting the expression of nuclear P65 and P50 and found that the expression levels of P65 and P50 in the Prdx6siRNA group were obviously higher than those in the NC-siRNA group, indicating that Prdx6 could suppress the nuclear translocation of P65 and P50. Our immunofluorescence results further confirmed these findings. However, further studies are required to clarify the mutual regulatory mechanism between Prdx6 and the NF-KB signaling pathway.

\section{Conclusions}

In our inflammation and oxidative stress injury model induced by $20 \% \mathrm{OLV}_{\text {after }}$ serum in vitro, downregulation of Prdx6 led to the activation of the NF-KB signaling pathway, which caused the 
subsequent overproduction of inflammatory cytokines and ROS. Pretreatment with curcumin restored Prdx 6 downregulation and inhibited NF-kB pathway activation by suppressing the nuclear translocation of P65 and P50. Curcumin protected against ALI not only by reducing the expression of proinflammatory and prooxidative factors but also by increasing the expression of anti-inflammatory and antioxidative factors. All these factors eventually reduce inflammation and oxidative stress damage induced by serum from patients undergoing OLV with thoracic surgery. Our findings offer new insights into the mechanism of $A L I$ induced by OLV, and the protective effect of curcumin provides a new reference for its prevention and treatment.

\section{Abbreviations}

OLV: One-lung ventilation; ALI: Acute lung injury; ARDS: Acute respiratory distress syndrome; Prdx6: Peroxiredoxin 6; NF-kB: Nuclear factor kappa B; Gpx: Glutathione peroxidase; aiPLA2: Calciumindependent phospholipase A2; LPCAT: Lysophosphatidylcholine acyltransferase; FBS: Fetal bovine serum; BSA: Bovine serum albumin; LPS: Lipopolysaccharide; VATS: Video-assisted thoracoscopic surgery; ROS: Reactive oxygen species; SOD: Superoxide dismutase; MDA: Malondialdehyde; $\mathrm{SpO}_{2}: \mathrm{Pulse}$ oxygen saturation; V/Q: Ventilation/perfusion; qRT-PCR: Quantitative reverse transcription PCR; ELISA: Enzyme-linked immunosorbent assay; DCFH-DA: Dichlorodihydrofluorescein diacetate.

\section{Declarations}

\section{Ethics approval and consent to participate}

The study protocol was approved by the ethics committee of SYSUCC in accordance with the Declaration of Helsinki. Written informed consent was obtained from all patients.

\section{Consent for publication}

Not applicable.

\section{Availability of data and materials}

The datasets used and/or analyzed during the current study are available from the corresponding author on reasonable request.

\section{Competing interests}

No conflicts of interest, financial or otherwise, are declared by the authors.

\section{Funding}

This work was supported by the National Natural Science Foundation of China (NSFC: 82070072). 
LHT, LWQ and CXJ designed the research. ZTH and CLH provided the clinical samples. TF, CLH, THY and ZWA provided support for the experimental techniques. TF provided data analysis support. LHT wrote the manuscript, and CXJ revised the paper. All authors read and approved the final manuscript.

\section{Acknowledgments}

Not applicable.

\section{References}

1. Licker M, Fauconnet $P$, Villiger $Y$, Tschopp JM: Acute lung injury and outcomes after thoracic surgery. Curr Opin Anaesthesiol 2009, 22:61-67.

2. Eichenbaum KD, Neustein SM: Acute lung injury after thoracic surgery. J Cardiothorac Vasc Anesth 2010, 24:681-690.

3. Alam N, Park BJ, Wilton A, Seshan VE, Bains MS, Downey RJ, Flores RM, Rizk N, Rusch VW, Amar D: Incidence and risk factors for lung injury after lung cancer resection. Ann Thorac Surg 2007, 84:1085-1091; discussion 1091.

4. Dulu A, Pastores SM, Park B, Riedel E, Rusch V, Halpern NA: Prevalence and mortality of acute lung injury and ARDS after lung resection. Chest 2006, 130:73-78.

5. YEN-TA LU PGH, TIMOTHY W. EVANS: <Ishemia-reperfusion lung injury contribution of ischemianeutrophils and hydrostatic pressure.pdf $>$. AM J PHYSIOL 1997, 273:46-54.

6. M Messent MJG, T W Evans: Pulmonary vascular reactivity and ischaemia-reperfusion injury in the rat.pdf. Clin Sci (lond) 1993, 85:71-75.

7. Williams EA, Quinlan GJ, Goldstraw P, Gothard JW, Evans TW: Postoperative lung injury and oxidative damage in patients undergoing pulmonary resection. Eur Respir J 1998, 11:1028-1034.

8. Liang D, Wen Z, Han W, Li W, Pan L, Zhang R: Curcumin protects against inflammation and lung injury in rats with acute pulmonary embolism with the involvement of microRNA-21/PTEN/NFkappaB axis. Mol Cell Biochem 2021, 476:2823-2835.

9. Li L, Huang Y, Zhang Z: Expression profile of miRNAs involved in the hepatoprotective effects of curcumin against oxidative stress in Nile tilapia. Aquat Toxicol 2021, 237:105896.

10. Sena-Junior AS, Aidar FJ, Oliveira ESAM, Estevam CDS, de Oliveira Carvalho CR, Lima FB, Dos Santos $\mathrm{JL}$, Marcal AC: Whether or Not the Effects of Curcuma longa Supplementation Are Associated with Physical Exercises in T1DM and T2DM: A Systematic Review. Nutrients 2020, 13.

11. Ashry AM, Hassan AM, Habiba MM, El-Zayat A, El-Sharnouby ME, Sewilam H, Dawood MAO: The Impact of Dietary Curcumin on the Growth Performance, Intestinal Antibacterial Capacity, and Haemato-Biochemical Parameters of Gilthead Seabream (Sparus aurata). Animals (Basel) 2021, 11.

12. Chen S, Liang H, Ji Y, Kou H, Zhang C, Shang G, Shang C, Song Z, Yang L, Liu L, et al: Curcumin Modulates the Crosstalk Between Macrophages and Bone Mesenchymal Stem Cells to Ameliorate Osteogenesis. Front Cell Dev Biol 2021, 9:634650. 
13. Shakeri A, Panahi Y, Johnston TP, Sahebkar A: Biological properties of metal complexes of curcumin. Biofactors 2019, 45:304-317.

14. Kareem A, Laxmi, Arshad M, Nami SA, Nishat N: Herbo-mineral based Schiff base ligand and its metal complexes: Synthesis, characterization, catalytic potential and biological applications. $J$ Photochem Photobiol B 2016, 160:163-171.

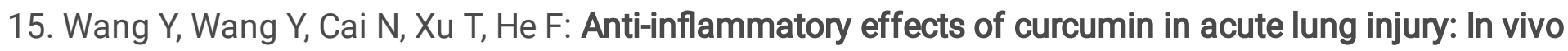
and in vitro experimental model studies. Int Immunopharmaco/ 2021, 96:107600.

16. Chen YQ, Chai YS, Xie K, Yu F, Wang CJ, Lin SH, Yang YZ, Xu F: Curcumin Promotes the Expression of IL-35 by Regulating Regulatory T Cell Differentiation and Restrains Uncontrolled Inflammation and Lung Injury in Mice. Inflammation 2020, 43:1913-1924.

17. Almatroodi SA, Alrumaihi F, Alsahli MA, Alhommrani MF, Khan A, Rahmani AH: Curcumin, an Active Constituent of Turmeric Spice: Implication in the Prevention of Lung Injury Induced by Benzo(a) Pyrene (BaP) in Rats. Molecules 2020, 25.

18. Vazquez-Medina JP, Tao JQ, Patel P, Bannitz-Fernandes R, Dodia C, Sorokina EM, Feinstein SI, Chatterjee S, Fisher AB: Genetic inactivation of the phospholipase A2 activity of peroxiredoxin 6 in mice protects against LPS-induced acute lung injury. Am J Physiol Lung Cell Mol Physiol 2019, 316:L656-L668.

19. Yang D, Song Y, Wang X, Sun J, Ben Y, An X, Tong L, Bi J, Wang X, Bai C: Deletion of peroxiredoxin 6 potentiates lipopolysaccharide-induced acute lung injury in mice. Crit Care Med 2011, 39:756-764.

20. Christian Zanza YL, Mirco Leo, Tatsiana Romenskaya, Francesco Franceschi: Practical Review of Mechanical Ventilation in Adults and Children in The Operating Room and Emergency Department.pdf. Rev Recent Clin Trials 2021.

21. Kozian A, Schilling T, Freden F, Maripuu E, Rocken C, Strang C, Hachenberg T, Hedenstierna G: Onelung ventilation induces hyperperfusion and alveolar damage in the ventilated lung: an experimental study. Br J Anaesth 2008, 100:549-559.

22. Kozian A, Schilling T, Rocken C, Breitling C, Hachenberg T, Hedenstierna G: Increased alveolar damage after mechanical ventilation in a porcine model of thoracic surgery. $J$ Cardiothorac Vasc Anesth 2010, 24:617-623.

23. Huang C, Wang Y, Li X, Ren L, Zhao J, Hu Y, Zhang L, Fan G, Xu J, Gu X, et al: Clinical features of patients infected with 2019 novel coronavirus in Wuhan, China. The Lancet 2020, 395:497-506.

24. Del Valle DM, Kim-Schulze S, Huang HH, Beckmann ND, Nirenberg S, Wang B, Lavin Y, Swartz TH, Madduri D, Stock $A$, et al: An inflammatory cytokine signature predicts COVID-19 severity and survival. Nat Med 2020, 26:1636-1643.

25. Misthos P, Katsaragakis S, Milingos N, Kakaris S, Sepsas E, Athanassiadi K, Theodorou D, Skottis I: Postresectional pulmonary oxidative stress in lung cancer patients. The role of one-lung ventilation. Eur J Cardiothorac Surg 2005, 27:379-382; discussion 382-373.

26. Grocott HP: Oxygen toxicity during one-lung ventilation: is it time to reevaluate our practice? Anesthesiol Clin 2008, 26:273-280, v. 
27. Stenger $\mathrm{C}$, Naves T, Verdier M, Ratinaud MH: The cell death response to the ROS inducer, cobalt chloride, in neuroblastoma cell lines according to p53 status. Int J Oncol 2011, 39:601-609.

28. Zuo L, Wijegunawardana D: Redox Role of ROS and Inflammation in Pulmonary Diseases. Adv Exp Med Biol 2021, 1304:187-204.

29. Wang X, Xu H, Guo M, Shen Y, Li P, Wang Z, Zhan M: The use of an oxidative stress scoring system in prognostic prediction for kidney renal clear cell carcinoma. Cancer Commun (Lond) 2021, 41:354357.

30. Jia G, Tan B, Ma J, Zhang L, Jin X, Li C: Prdx6 Upregulation by Curcumin Attenuates Ischemic Oxidative Damage via SP1 in Rats after Stroke. Biomed Res Int 2017, 2017:6597401.

31. Chhunchha B, Fatma N, Kubo E, Rai P, Singh SP, Singh DP: Curcumin abates hypoxia-induced oxidative stress based-ER stress-mediated cell death in mouse hippocampal cells (HT22) by controlling Prdx6 and NF-kappaB regulation. Am J Physiol Cell Physiol 2013, 304:C636-655.

32. Chhunchha B, Fatma N, Bhargavan B, Kubo E, Kumar A, Singh DP: Specificity protein, Sp1-mediated increased expression of Prdx6 as a curcumin-induced antioxidant defense in lens epithelial cells against oxidative stress. Cell Death Dis 2011, 2:e234.

33. Liao J, Zhang Y, Chen X, Zhang J: The Roles of Peroxiredoxin 6 in Brain Diseases. Mol Neurobiol 2021.

34. Fatma N, Singh P, Chhunchha B, Kubo E, Shinohara T, Bhargavan B, Singh DP: Deficiency of Prdx6 in lens epithelial cells induces ER stress response-mediated impaired homeostasis and apoptosis. Am J Physiol Cell Physiol 2011, 301:C954-967.

35. Fisher AB, Dodia C, Chatterjee S: A Peptide Inhibitor of Peroxiredoxin 6 Phospholipase A2 Activity Significantly Protects against Lung Injury in a Mouse Model of Ventilator Induced Lung Injury (VILI). Antioxidants (Basel) 2021, 10.

36. Wang X, An X, Wang X, Hu X, Bi J, Tong L, Yang D, Song Y, Bai C: Peroxiredoxin 6 knockout aggravates cecal ligation and puncture-induced acute lung injury. Int Immunopharmaco/ 2019, 68:252-258.

37. Hans Hacker MK: Regulation and function of IKK and IKK related kinases.pdf. Sci STKE 2006, 2006:13.

38. Sharapov MG, Glushkova OV, Parfenyuk SB, Gudkov SV, Lunin SM, Novoselova EG: The role of TLR4/NF-kappaB signaling in the radioprotective effects of exogenous Prdx6. Arch Biochem Biophys 2021, 702:108830.

39. Min Y, Wi SM, Shin D, Chun E, Lee KY: Peroxiredoxin-6 Negatively Regulates Bactericidal Activity and NF-kappaB Activity by Interrupting TRAF6-ECSIT Complex. Front Cell Infect Microbiol 2017, 7:94.

\section{Figures}



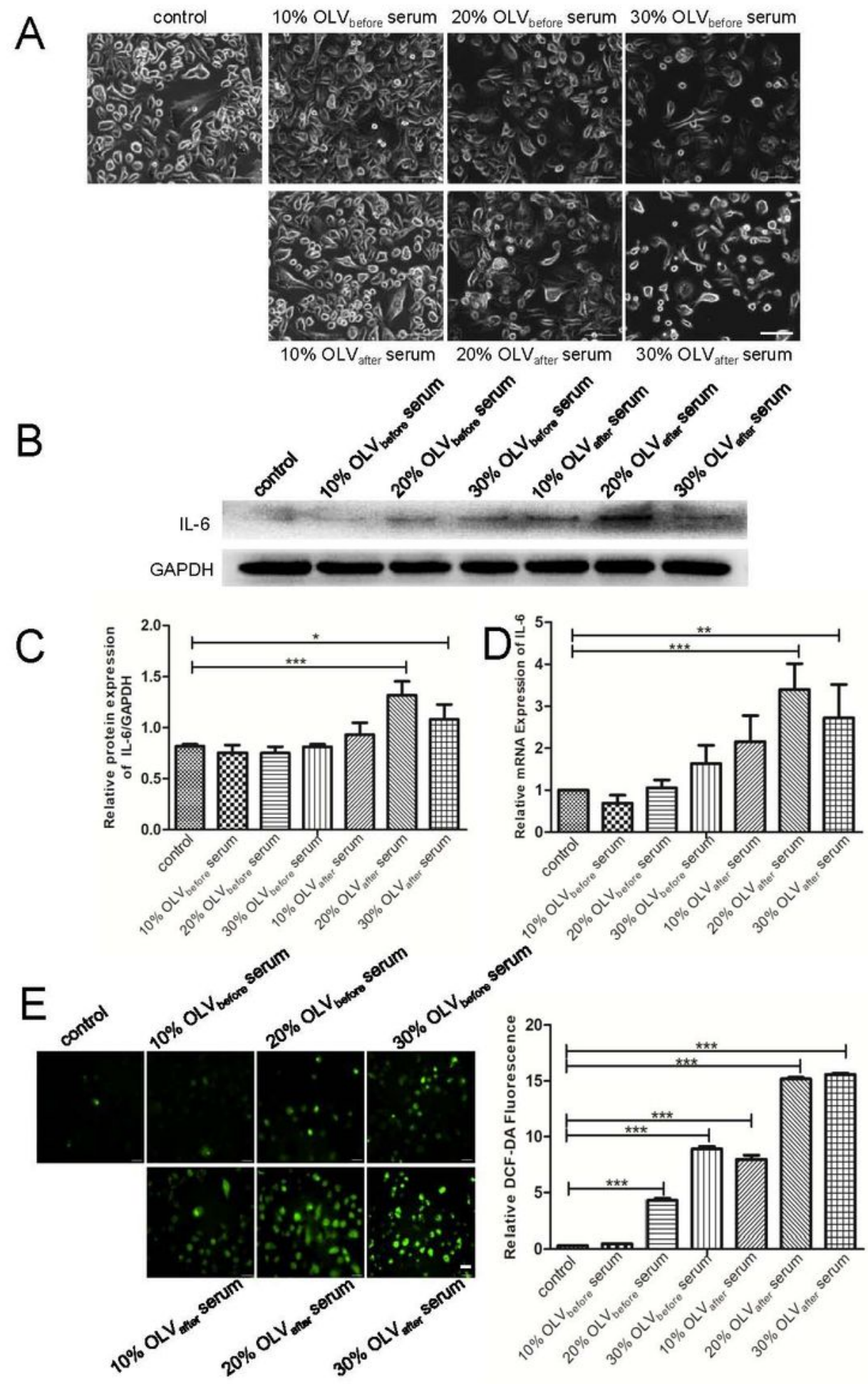

\section{Figure 1}

Twenty percent OLVafter serum induced the most obvious inflammation and oxidative stress in A549 cells. (A) Representative image of cell morphology after treatment with different concentrations of serum. Scale bar $=100 \mu \mathrm{m}$. (B) Western blot analysis images of IL-6 in each group. (C) The relative protein levels of IL-6 were calculated. (D) qRT-PCR analysis of IL-6 in each group. (E) ROS levels were measured by DCFH-DA staining, and the relative DCF-DA fluorescence intensities were normalized and summarized. 
Scale bar $=100 \mu \mathrm{m}$. The results are shown as the means \pm SD of 3 individual experiments. ${ }^{*}, P<0.05 ; \star \star *$ $\mathrm{P}<0.01 ; * \star * \mathrm{P}<0.001$.
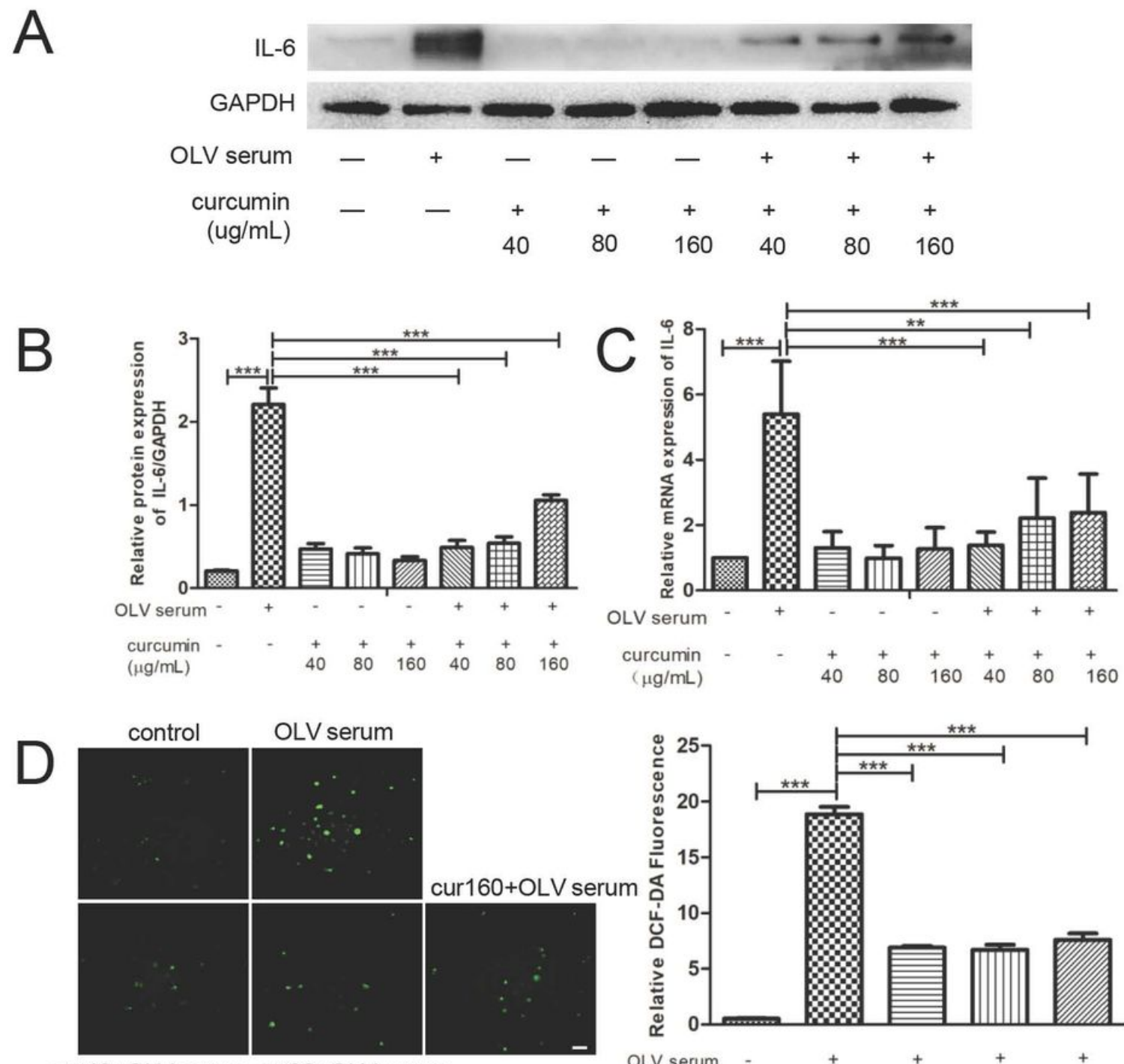

cur40+OLV serum cur80+OLV serum
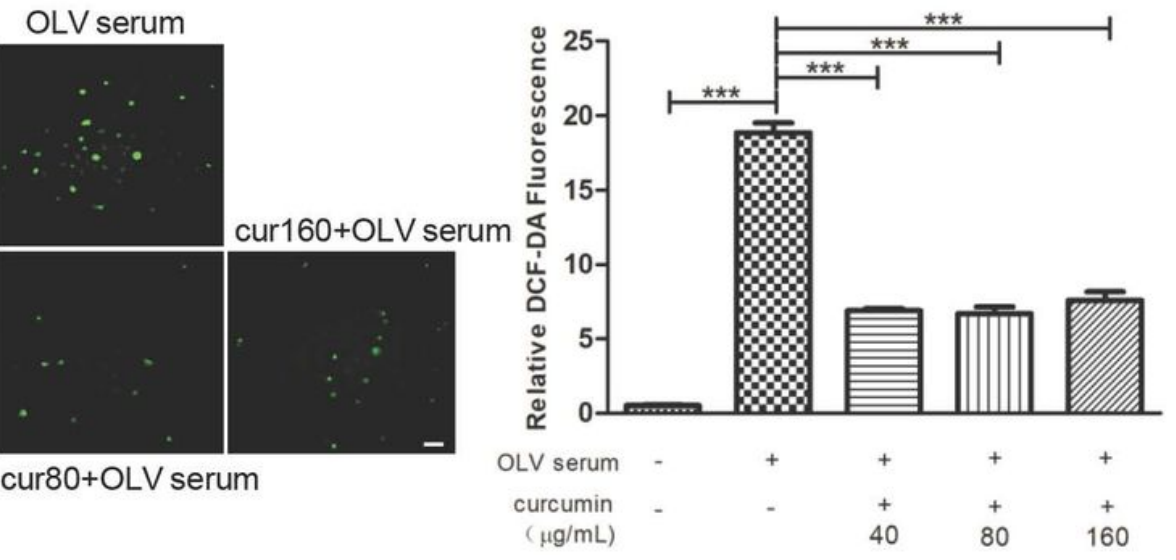

Figure 2

Different concentrations of curcumin alleviate inflammation and oxidative stress in A549 cells. (A) Western blot analysis images of IL-6 in each group. (B) The relative protein levels of IL- 6 were calculated. (C) qRT-PCR analysis of IL-6 in each group. (D) ROS levels measured by DCFH-DA staining and the relative DCF-DA fluorescence intensities were normalized and summarized. Scale bar $=50 \mu \mathrm{m}$. The results are shown as the means $\pm S D$ of 3 individual experiments. ${ }^{*} P<0.05 ; * \star P<0.01 ; * \star \star ~ P<0.001$. 


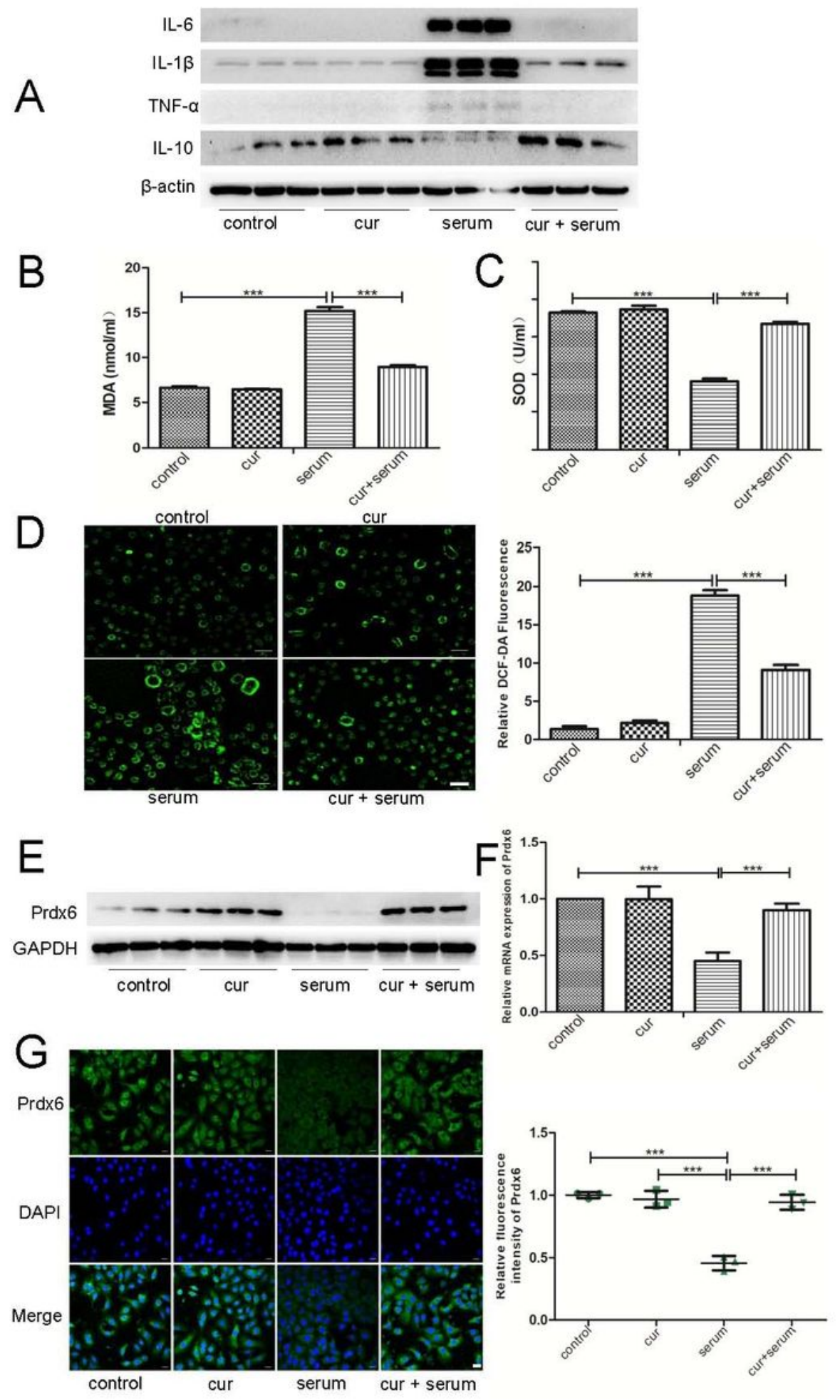

Figure 3

The protective effect of curcumin on OLV-induced ALI is related to Prdx6. (A) Western blot analysis of representative inflammatory factor levels, among which IL-6, IL-1 $\beta$, and TNF- $\alpha$ are the classic proinflammatory factors and IL-10 is the anti-inflammatory factor in each group. (B) ELISA analysis of MDA levels in each group. (C) ELISA analysis of SOD levels in each group. (D) ROS levels were measured by DCFH-DA staining in each group. Scale bar $=100 \mu \mathrm{m}$. (E) Western blot analysis images of Prdx6 in 
each group. (F) qRT-PCR analysis of Prdx6 in each group. (G) Immunofluorescence analysis of Prdx6 in each group. DAPI is shown in blue and represents the nucleus. Scale bar $=100 \mu \mathrm{m}$. In the control group (control): cells were pretreated with DMSO vehicle alone for $2 \mathrm{~h}$, then changed to serum-free medium for $48 \mathrm{~h}$; in curcumin group (cur): cells were pretreated with $40 \mu \mathrm{g} / \mathrm{ml}$ curcumin + DMSO for $2 \mathrm{~h}$, then changed to serum-free medium for $48 \mathrm{~h}$; in the serum group (serum): the cells were intervened with $20 \%$ OLVafter serum for $48 \mathrm{~h}$; in the curcumin + serum group (cur + serum): the cells were first pretreated with $40 \mu \mathrm{g} / \mathrm{ml}$ curcumin and DMSO, then changed to $20 \%$ OLVafter serum for $48 \mathrm{~h}$. The results are shown as the means \pm SD of 3 individual experiments. ${ }^{*} P<0.05 ; * \star P<0.01 ; * \star \star ~ P<0.001$. 


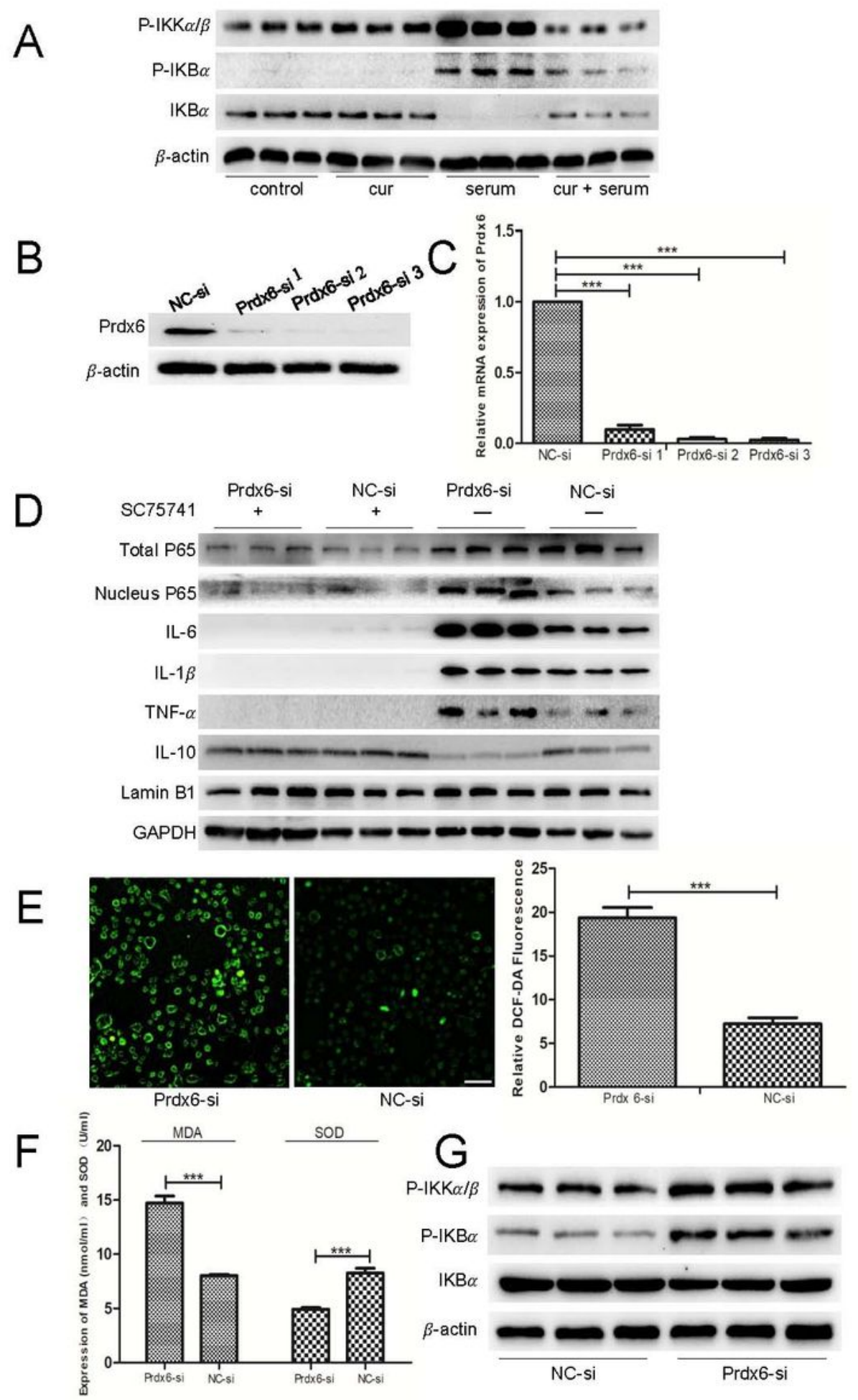

Figure 4

Prdx6 mediated the protective function of curcumin in OLV-induced ALI by regulating the NF-KB pathway. (A) The levels of NF-kB signaling markers in each group were determined via Western blot. (B) Western blot analysis of Prdx6 expression in A549 cells transfected with either Prdx6-siRNA or NC-siRNA. (C) qRTPCR analysis of Prdx6 expression in A549 cells transfected with either Prdx6-siRNA or NC-siRNA. (D) The expression of representative inflammatory factors and P65 in Prdx6-siRNA- or NC-siRNA-transfected cells 
with or without SC75741. (E) ROS levels were measured by DCFH-DA staining in cells transfected with either Prdx6-siRNA or NC-siRNA. Scale bar $=100 \mu \mathrm{m}$. (F) ELISA analysis of oxidative stress factor levels (MDA and SOD) in cells transfected with either Prdx6-siRNA or NC-siRNA. (G) The protein levels of NF-KB signaling markers in cells transfected with either Prdx6-siRNA or NC-siRNA. In the control group (control): the cells were pretreated with DMSO vehicle alone for $2 \mathrm{~h}$, then changed to serum-free medium for $48 \mathrm{~h}$; curcumin group (cur): the cells were pretreated with $40 \mu \mathrm{g} / \mathrm{ml}$ curcumin + DMSO for $2 \mathrm{~h}$, then changed to serum-free medium for $48 \mathrm{~h}$; serum group (serum): the cells were intervened with $20 \%$ OLVafter serum for $48 \mathrm{~h}$; curcumin + serum group (cur + serum): the cells were first pretreated with $40 \mu \mathrm{g} / \mathrm{ml}$ curcumin and DMSO, then changed to $20 \%$ OLVafter serum for $48 \mathrm{~h}$. The results are shown as the means \pm SD of 3 individual experiments. ${ }^{\star} \mathrm{P}<0.05 ; * \star \mathrm{P}<0.01 ; * \star * \mathrm{P}<0.001$.

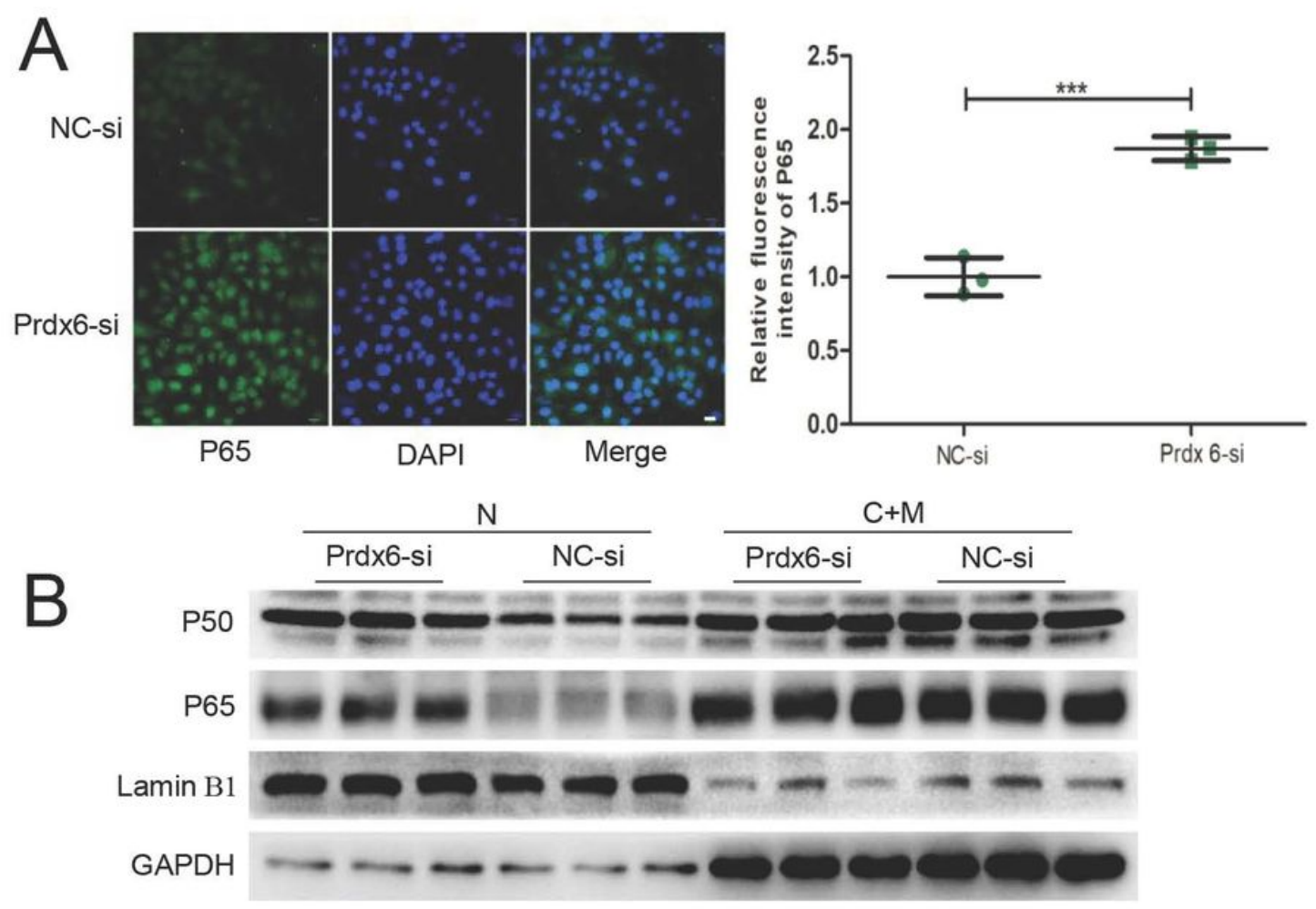

\section{Figure 5}

Prdx6 inhibits activation of the NF-kB signaling pathway by suppressing the nuclear translocation of P65. (A) Immunofluorescence analysis of P65 in cells transfected with either Prdx6-siRNA or NC-siRNA. DAPI is shown in blue and represents the nucleus. Scale bar $=100 \mu \mathrm{m}$. (B) Nuclear (N) and cytosolic/membrane $(\mathrm{C}+\mathrm{M})$ proteins of the NF-KB pathway in Prdx6-siRNA- or NC-siRNA-transfected cells. The cells in all groups were first pretreated with $40 \mu \mathrm{g} / \mathrm{ml}$ curcumin and DMSO and then changed to $20 \%$ OLVafter serum for $48 \mathrm{~h}$. The results are shown as the means \pm SD of 3 individual experiments. ${ }^{*} P<0.05$; $* *$ $\mathrm{P}<0.01 ; * \star \star \mathrm{P}<0.001$. 